\title{
Quantitative evaluation of deep convolutional neural network-based image denoising for low-dose computed tomography
}

Keisuke Usui ${ }^{1,2^{*}}$, Koichi Ogawa ${ }^{3}$, Masami Goto ${ }^{1}$, Yasuaki Sakano ${ }^{1}$, Shinsuke Kyougoku ${ }^{1}$ and Hiroyuki Daida ${ }^{1}$

\begin{abstract}
To minimize radiation risk, dose reduction is important in the diagnostic and therapeutic applications of computed tomography (CT). However, image noise degrades image quality owing to the reduced X-ray dose and a possible unacceptably reduced diagnostic performance. Deep learning approaches with convolutional neural networks (CNNs) have been proposed for natural image denoising; however, these approaches might introduce image blurring or loss of original gradients. The aim of this study was to compare the dose-dependent properties of a CNN-based denoising method for low-dose CT with those of other noise-reduction methods on unique CT noisesimulation images. To simulate a low-dose CT image, a Poisson noise distribution was introduced to normal-dose images while convoluting the CT unit-specific modulation transfer function. An abdominal CT of 100 images obtained from a public database was adopted, and simulated dose-reduction images were created from the original dose at equal 10-step dose-reduction intervals with a final dose of 1/100. These images were denoised using the denoising network structure of CNN (DnCNN) as the general CNN model and for transfer learning. To evaluate the image quality, image similarities determined by the structural similarity index (SSIM) and peak signalto-noise ratio (PSNR) were calculated for the denoised images. Significantly better denoising, in terms of SSIM and PSNR, was achieved by the DnCNN than by other image denoising methods, especially at the ultra-low-dose levels used to generate the $10 \%$ and $5 \%$ dose-equivalent images. Moreover, the developed CNN model can eliminate noise and maintain image sharpness at these dose levels and improve SSIM by approximately $10 \%$ from that of the original method. In contrast, under small dose-reduction conditions, this model also led to excessive smoothing of the images. In quantitative evaluations, the CNN denoising method improved the low-dose CT and prevented oversmoothing by tailoring the CNN model.
\end{abstract}

Keywords: Deep learning, Convolutional neural network, Low-dose computed tomography, Denoising, Image quality

\footnotetext{
* Correspondence: k-usui@juntendo.ac.jp

${ }^{1}$ Department of Radiological Technology, Faculty of Health Science,

Juntendo University, Tokyo 113-8421, Japan

${ }^{2}$ Department of Radiation Oncology, Faculty of Medicine, Juntendo

University, Tokyo 113-8421, Japan

Full list of author information is available at the end of the article
}

\section{Springer Open}

(c) The Author(s). 2021 Open Access This article is licensed under a Creative Commons Attribution 4.0 International License, which permits use, sharing, adaptation, distribution and reproduction in any medium or format, as long as you give appropriate credit to the original author(s) and the source, provide a link to the Creative Commons licence, and indicate if changes were made. The images or other third party material in this article are included in the article's Creative Commons licence, unless indicated otherwise in a credit line to the material. If material is not included in the article's Creative Commons licence and your intended use is not permitted by statutory regulation or exceeds the permitted use, you will need to obtain permission directly from the copyright holder. To view a copy of this licence, visit http://creativecommons.org/licenses/by/4.0/. 


\section{Introduction}

Computed tomography (CT) is widely used for repetitive screening diagnostic scans, such as scans for cancer, lung nodules, and bleeding internal organs. Highexposure scans can cause patients to suffer from several biological effects that increase the risk of cancer $[1,2]$. To minimize exposure doses, reducing the number of $\mathrm{X}$ ray photons via tube-current modulation is a viable solution. However, this method is limited because low-dose CT triggers image-quality degradation. Hence, lower doses of X-ray photons provide noisier reconstructed CT images, which may reduce the diagnostic performance to an unacceptable level [3].

To address this image degradation, several techniques have been proposed to improve the quality of low-dose CT images. Sinogram domain filtration and iterative reconstruction methods combine the statistical properties of the data in the image domain and projection space to optimize the objective function. Although these methods can eliminate image noise, they depend on the specifications of the manufacturer, thereby limiting their clinical applications. Computational costs are also required; hence, the reconstruction process is relatively slow [4, 5]. Moreover, image-space denoising methods, such as median, Gaussian, and Wiener filters, do not require projection data and aim to reduce image noise without requiring an understanding of the structures of interest. Therefore, they are exposed to the risk of either generating new image artifacts or losing original structural information during post-processing, which limit their clinical applications [6].

Deep learning approaches have been widely used for image denoising, and convolutional neural networks (CNNs), which are based on extensive data and powerful graphics-processing units, have achieved substantial success $[7,8]$. Several CNN-based methods have been proposed for natural image denoising and low-dose CT. However, these CNNs are often inhibited by disappearing gradients and the introduction of image blurring, which leads to difficulties in training [9]. A denoising network (Dn), known as DnCNN, incorporates residual learning and batch normalization (BN), and can yield better improvements for Gaussian denoising tasks with unknown noise. In this technique, residual learning can be realized by improving the training efficiency when the input and output are close to each other, such as a noise image [10]. Moreover, BN facilitates the application of significantly higher learning rates by stabilizing the training process, which can address the vanishing gradient challenge in these deeper learning processes [11].

However, this method has been demonstrated to be inferior to more realistic and complicated noises [10, 12]. Several CNN-based methods have been evaluated for clinical low-dose CT denoising. Chen et al. [13] demonstrated the potential of a CNN-based framework for artifact reduction and structure preservation in lowdose CT imaging. Deep learning algorithms that adopt residual encoder-decoder networks have been evaluated and validated for CT denoising, and these networks have exhibited significant potential for noise suppression, structural preservation, and lesion detection at a high computational speed [14]. However, the dependence of the incident dose reduction in the $\mathrm{CNN}$-based denoising method has not been elucidated, and dose reduction levels for the effective operation of the CNN model need to be validated.

In this study, to better understand the dose-dependent properties of the CNN-based method, DnCNN was implemented to denoise low-dose CT images, and compared the image quality with that of other noise-reduction methods on unique CT noise simulation images. Collecting real human images obtained at various incident dose levels to train a deep learning model significantly increases the cost of the learning process. To address this challenge, the aim of this study was to simulate virtual low-dose images at several dose-reduction levels, and apply physical evaluation metrics of image quality to quantitatively compare the noise-reduction performance of different denoising methods on simulated images.

\section{Methods \\ Network architecture}

The network structure of the DnCNN was adopted as the general CNN model [10]. This model was pretrained on 400 Gy-level images with a Gaussian noise level $\sigma=25$ for natural image denoising. The input image size for training was $50 \times 50$ pixels, the convolution layers were set to 20 , and 64 convolutional filters were used at a size of $3 \times 3$ pixels to generate the feature maps. Residual learning and $\mathrm{BN}$ were used to enhance denoising performance and learning speed. Moreover, defined as the positive part of its value, rectified linear units (ReLU) were adopted for faster training of deep neural networks. DnCNN learns residual map data and generates a noise-reduced image, which can yield more efficient training and accurate results within very deep networks. BN was applied between the convolution filters, and ReLU was used in all middle layers to enable higher learning rates with the normalization of each sub-sample set. The architecture of the DnCNN network is illustrated in Fig. 1.

\section{Transfer learning}

The concept of the proposed transfer learning is to update the pretrained model of the DnCNN for adapting CT-specific dose-reduction images. In this study, $100 \mathrm{ab}-$ dominal CT images obtained with a sufficient original dose were adopted for transfer learning. These CT images were obtained from a publicly available dataset 


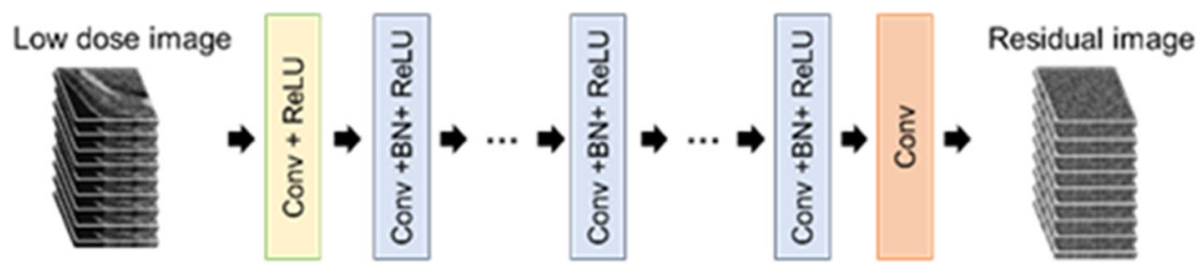

Fig. 1 Overall architecture of the DnCNN with simulated-noise images. This network learns residual mapping for noise image. The main modules include convolutional filtering, ReLU and BN

(The Cancer Imaging Archive), which is an open-access information resource created by the US National Cancer Institute [15]. Simulated dose-reduction images were generated within these abdominal images, with a 10 -step dose reduction rate from the original dose to $1 / 100$. Therefore, the number of training images in the proposed transfer learning was 1000 . Subsequently, these images were selected in two cases, and 5\% of the data were used to validate the denoising performance during the training. Training images were cropped in $350 \times 250$ pixels $\left(350 \times 500 \mathrm{~mm}^{2}\right)$ in the center of the image and randomly divided into small patches of $50 \times 50$ pixels, with 15 patches per image. Furthermore, the input image was rotated randomly from $0^{\circ}$ to $90^{\circ}$, horizontally. These learning methods can prevent overfitting using training datasets. The Adam optimizer was used for training at a learning rate of $10^{-3}$, and the networks were trained in 150 epochs. A Quadro RTX 5000 GPU graphics processor (NVIDIA Corporation) was adopted for training and evaluation. The total training time was approximately $10 \mathrm{~h}$.

\section{CT noise-simulation images}

The dominant $\mathrm{CT}$ noise statistics in X-ray reduction are known to follow a Poisson distribution. Therefore, to simulate a low-dose CT image, a Poisson noise distribution was introduced while convoluting the CT unitspecific modulation transfer function (MTF) [16]. Figure 2 presents the MTF curve applied during the noise simulation process. By adding these simulated CT specific noises, statistical pixel value deviation images were created according to the incident dose reduction levels. Here, simulated noise were created with standard deviations of $1.3,2$, 10 , and 20 times the original image to create $75 \%, 50 \%$, $10 \%$, and $5 \%$ dose-equivalent images. Accordingly, unique reduced-dose images reflecting the $\mathrm{CT}$ unit noise characteristics were created for this denoising study.

\section{Evaluation of image quality}

To evaluate the image quality of denoised images using DnCNN, and compare its performance with that of other noise-reduction methods, the CT value intensity was measured, and the structural similarity index (SSIM)

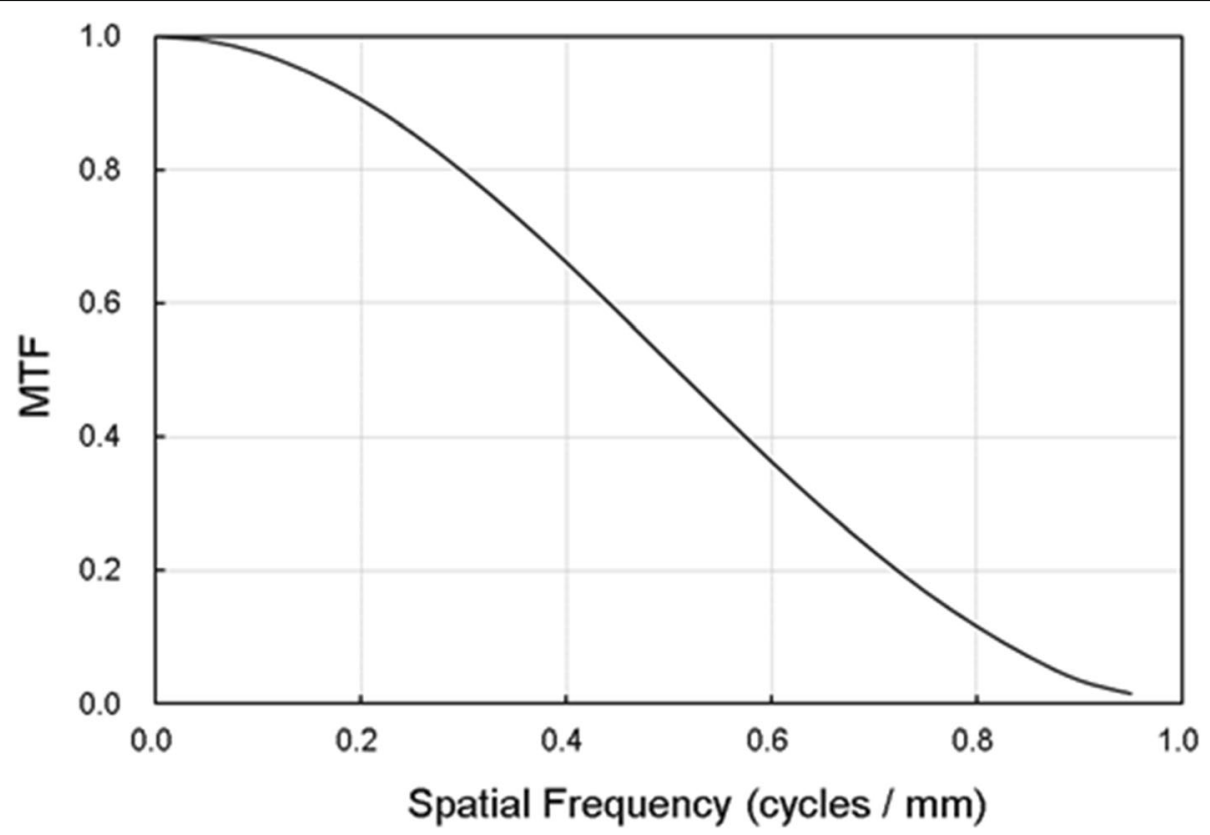

Fig. 2 CT unit-specific modulation transfer function 
and peak signal-to-noise ratio (PSNR) were calculated for the denoised images [17]. The SSIM was calculated as follows:

$$
\operatorname{SSIM}(x, y)=\frac{\left(2 \mu_{x} \mu_{y}+C_{1}\right)\left(2 \sigma_{x y}+C_{2}\right)}{\left(\mu^{2}{ }_{x}+\mu^{2}{ }_{y}+C_{1}\right)\left(\sigma^{2}{ }_{x}+\sigma^{2}{ }_{y}+C_{2}\right)}
$$

where $\mu, \sigma, C$, and $x, y$ represent the signal mean, variance, $C$ regularization constant, and each evaluation image, respectively. Furthermore, the PSNR was calculated as:

$$
\begin{aligned}
& M S E=\frac{1}{M \times N} \sum_{i=1}^{M} \sum_{j=1}^{N}[x(i, j)-y(i, j)]^{2}, \\
& P S N R=10 \log _{10} \frac{\max |x(i, j)|^{2}}{M S E}
\end{aligned}
$$

PSNR is the maximum value in the input image data $[x(i, j)]$ divided by the mean squared error (MSE) between image $x$ (denoised low-dose image) and image $y$ (original full-dose image). In addition, $M$ and $N$ represent the width and height of the images, respectively.

In this study, the performance of $\mathrm{DnCNN}$ was compared with other noise-reduction methods using median, Gaussian, and Wiener filters. In the median filter method, the output value is the median value of the $3 \times$ 3-neighboring pixels. In the Gaussian filter method, an isotropic Gaussian smoothing kernel with a standard deviation of 1.0 is applied to a two-dimensional image. In the Wiener filter method, smoothing kernels in the mean and variance of $5 \times 5$-neighboring pixels were adopted in the denoising process. Moreover, post-transfer-learned DnCNN was compared with these noise-reduction methods, including the original DnCNN. To compare image quality by adopting the indices of PSNR and SSIM, 10 abdominal CT images composed of $350 \times 250$ pixels $\left(350 \times 250 \mathrm{~mm}^{2}\right)$ with 3 $\mathrm{mm}$ slice thicknesses were used for the quantitative evaluation. These CT images were obtained from a publicly available dataset, from the Cancer Imaging Archive [13]. The simulated noise images were created in $75 \%, 50 \%, 10 \%$, and $5 \%$ equivalent doses relative to the original dose. The difference between the results of each noise reduction method and the original DnCNN method was considered statistically significant (two-tailed t-test, $p<0.05$ ).

\section{Results \\ Denoised images}

Figure 3 presents one of the results of the denoised images obtained using each filtering and CNN-based method. Figure $3 \mathrm{~b}$ compares the enlarged images of one region (indicated by the red rectangle) in Fig. 3a. Moreover, a vertical CT value profile of each image with equivalent dose levels of 50\% and 10\% are plotted in Fig. 4.

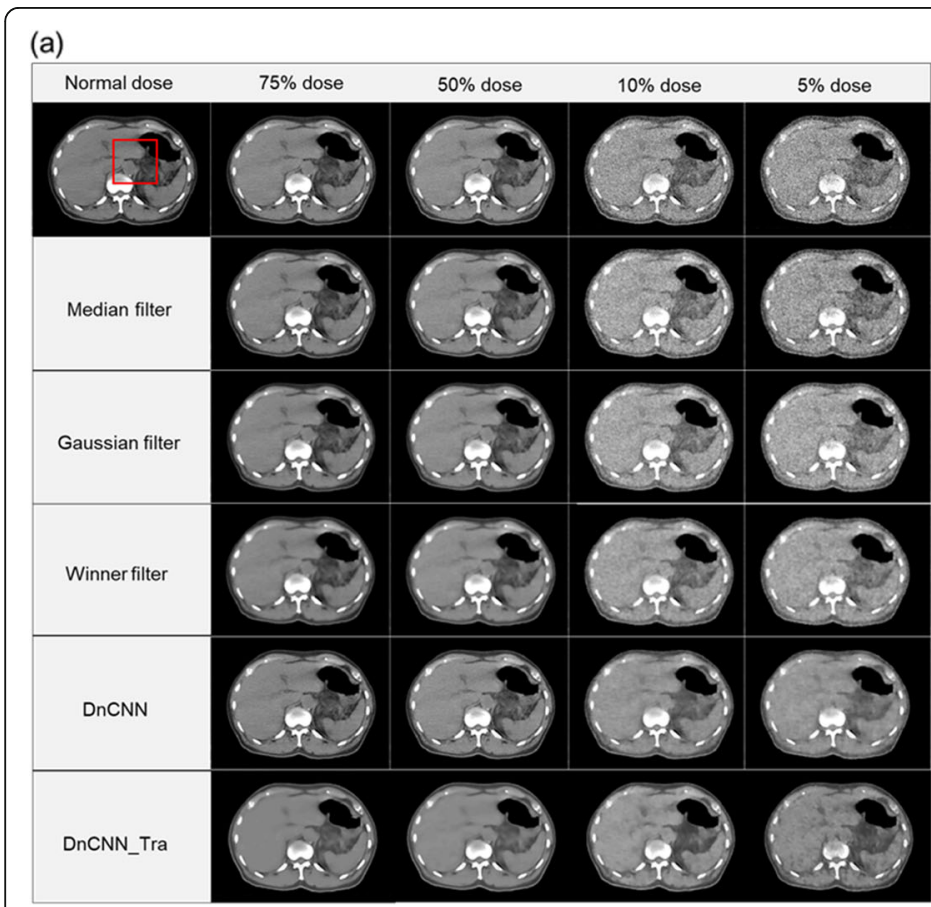

(b)

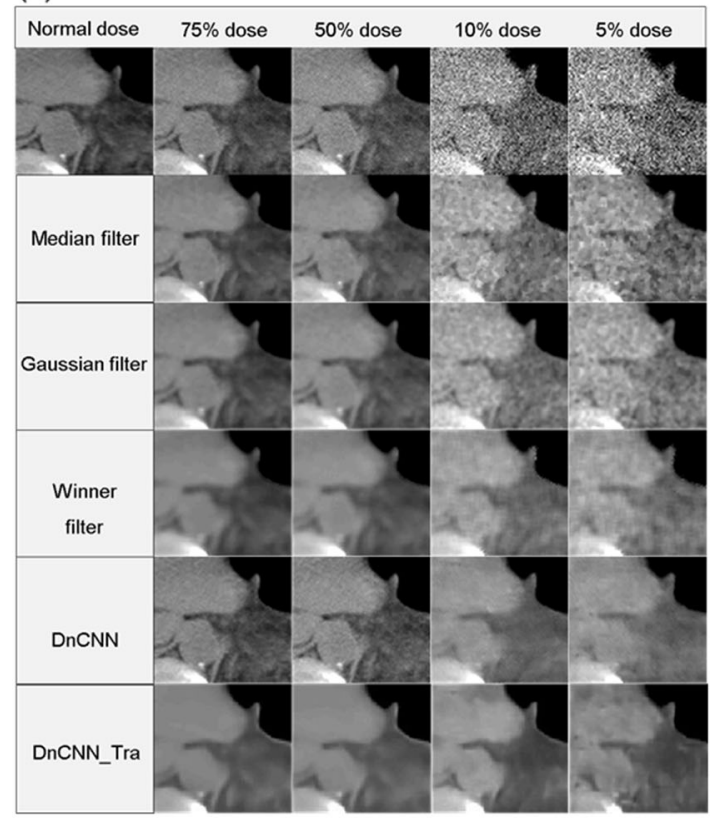

Fig. 3 Denoised image with contaminating noise artifacts, which was simulated to minimize dose exposure in the image at four different dose levels. a Results of noise reduction in the entire abdominal image; $\mathbf{b}$ Enlarged images of the region indicated by the red region of interest; all images are presented with the same window width and level. Median, Gaussian, Wiener, original DnCNN, and optimized DnCNN (DnCNN_Tra) techniques were applied for noise reduction at each dose-reduction level 

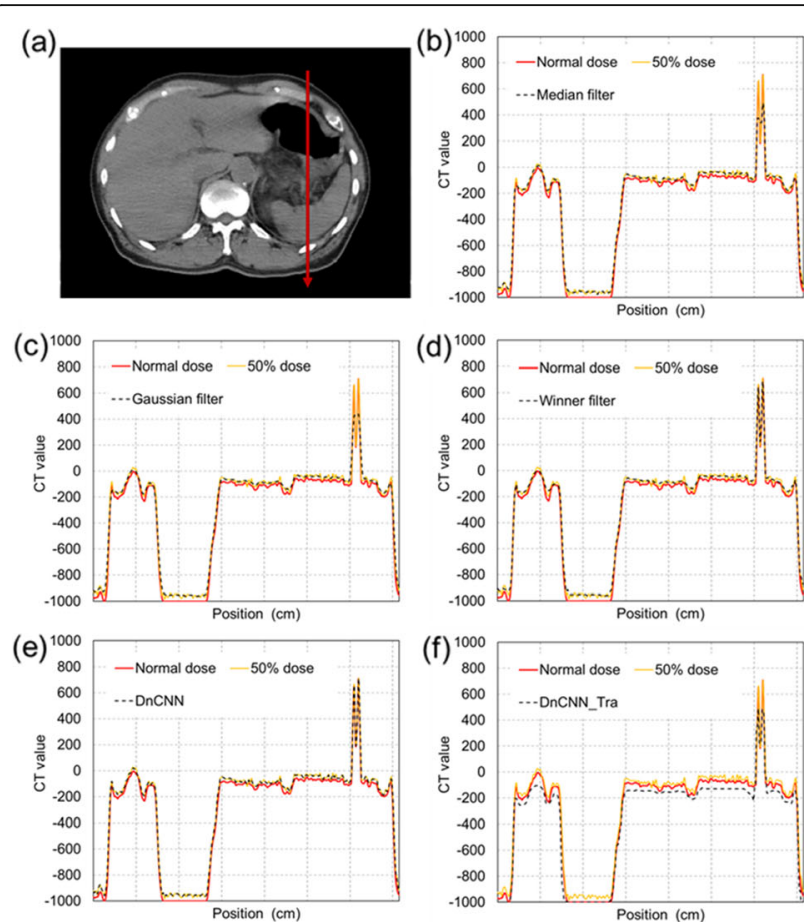

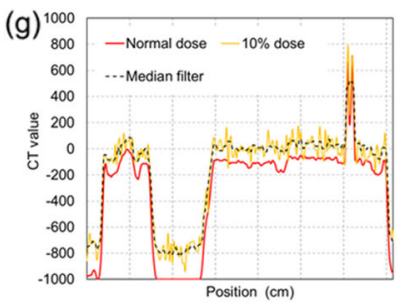

(i)
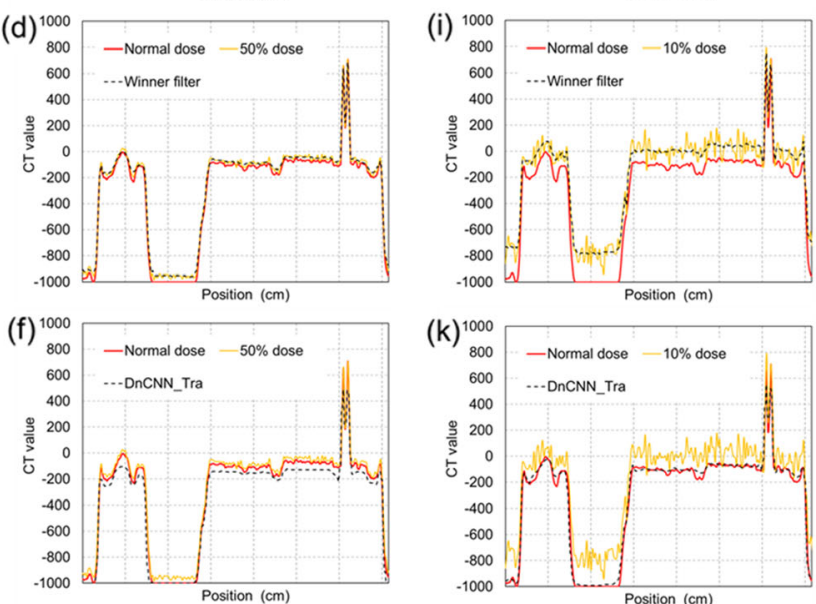

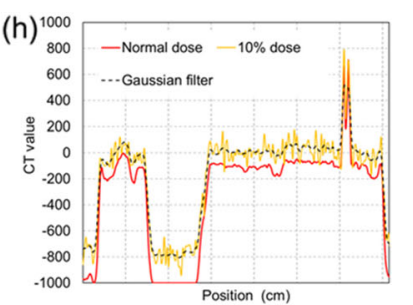

(j) 10000

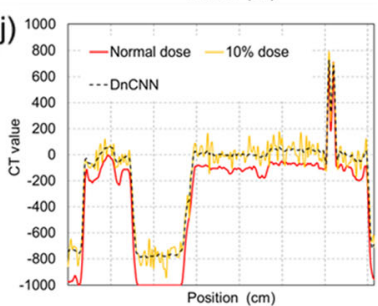

Fig. 4 Comparison of the vertical profiles in each image. a Profile position; b-f $C T$ value profiles along the red arrow in (a) with each noise reduction method in the $50 \%$ dose equivalent image; $\mathbf{g - k}$ CT value profiles along the same line in the $10 \%$ dose equivalent image. Red line depicts the normal dose image, yellow lines represent the dose reduction images, and each black dashed line denotes the denoised images by the Median, Gaussian, Winner, original DnCNN and DnCNN_Tra techniques, respectively

In these results, DnCNN_Tra represents the application of transfer learning for the original DnCNN model. Regarding the ultra-low-dose levels (equivalent to $10 \%$ and $5 \%$ doses), significant image degradation was triggered by the simulating the reduction in incident photons. The image quality improvement obtained by the filter-denoising methods at ultra-low doses was insufficient. In contrast, it was also observed that the $\mathrm{CNN}$-based methods effectively reduced the noise at these ultra-low doses. Figure 5 presents a comparison of visual inspection via denoising with the DnCNN method. In the results of the original DnCNN method, although the noise had been eliminated, image blurring was introduced at ultra-low-dose levels. Conversely, denoising using the DnCNN_Tra was adopted to preserve the image edge sharpness, and the hepatic sickle mesentery was visualized, as depicted by the white arrows in Fig. 5. Moreover, the profile of the CT value in the $10 \%$ dose equivalent image was similar to that of the normal dose image, as illustrated in Fig. 4k. Nevertheless, excessive smoothing at dosereduction levels of $75 \%$ and $50 \%$, and inaccurate blackout regions at dose-reduction levels of $5 \%$ were observed, as indicated by the red arrows in Fig. 5 .

\section{Quantitative evaluation of image quality}

In Figs. 5 and 6, the image qualities of the original DnCNN and DnCNN_Tra methods were compared in terms of SSIM and PSNR, respectively, over different dose-reduction levels among noise-suppression filters. For the SSIM values, the DnCNN method tended to provide improvements at all dose reduction levels. However, no significant difference was observed with respect to the results of the Gaussian filter method. In contrast, PSNR results were significantly improved in the $75 \%$ and $50 \%$ equivalent-dose images when compared with the results of the Gaussian filter method. However, at ultralow-dose levels, the DnCNN method could not obtain significantly higher PSNR values than the other noisereduction methods. For DnCNN_Tra, the SSIM was improved by approximately $10 \%$ relative to that of the original DnCNN method in the 5\% and $10 \%$ doseequivalent images. In contrast, in the results for the $75 \%$ and 50\% dose-equivalent images, SSIM and PSNR were significantly degraded relative to the results for the original DnCNN method.

\section{Discussion}

In this study, the ability of general pre-trained $\mathrm{CNN}$ image denoising to discard noise triggered by the lack of incident photons in CT images, was assessed. For diagnostic and radiation therapy purposes, denoised images should exhibit sufficient quality to facilitate clinically sound decisions. Therefore, the quantitative evaluation 


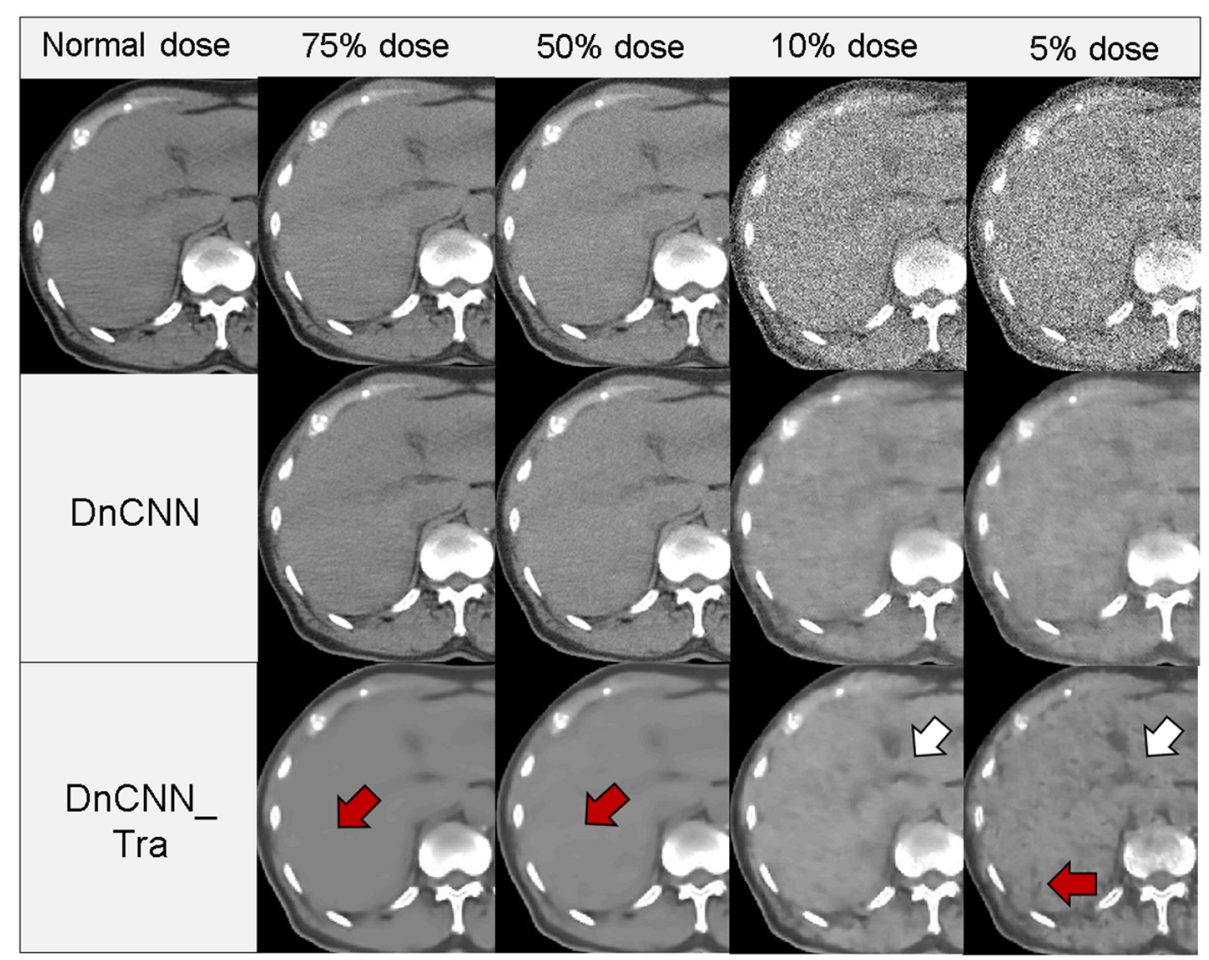

Fig. 5 Comparison of the visual inspection by denoising, between the original DnCNN and trained DnCNN methods. White arrows depict the hepatic sickle mesentery, which can be visualized by the denoising effect using the trained DnCNN method. Conversely, red arrows represent excessive smoothing triggered by the denoising

of image quality is based on its similarity to normal dose images when comparing noise-reduction methods.

The denoising performance in terms of image SSIM and PSNR was significantly better for DnCNN than for the other image-space denoising methods. In the results presented in Fig. 3, the DnCNN method effectively reduces noise at all dose reduction levels. The results of the image-space filtering methods at ultra-low levels of $10 \%$ and $5 \%$ dose equivalents were insufficient. These results were triggered by the extremely large deviation in the image pixel values, thus suggesting the limitations of denoising using a reconstructed image space.

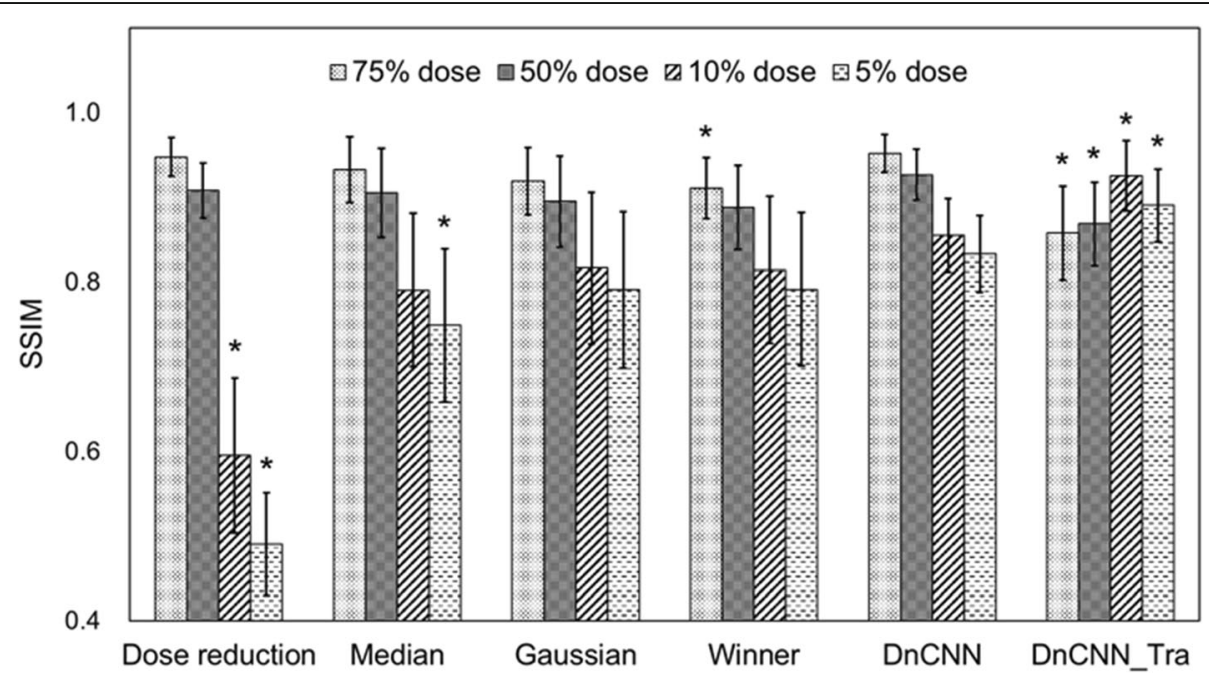

Fig. 6 SSIM results for different noise-reduction methods for each dose-reduction simulated image. ${ }^{*}$ indicates $p<0.05$, which represents a significant difference in the SSIM value relative to the results of the original DnCNN method 
As illustrated in Fig. 6, the DnCNN method tended to increase the SSIM value at all dose levels; therefore, an improvement in the overall image similarity is expected. Although the DnCNN model was pretrained on 400 grayscale images and a Gaussian noise level of 25 for natural image denoising [10], its potential effectiveness for denoising low-dose CT was also validated in our study. However, there were no significant differences in the SSIM values between the results of the DnCNN and Gaussian methods. A virtual dose-reduced image was simulated according to Poisson distribution. Accordingly, the Gaussian filter method was effective in removing noise in this study. In contrast, because the DnCNN model also learned a Gaussian distribution, effective results were obtained when removing noise statistics from low-dose CT images.

The results in Fig. 7 indicate that the DnCNN method realizes significantly better results than those of the Gaussian model at the $75 \%$ and $50 \%$ dose-equivalent levels. However, at ultra-low-dose levels, no significant improvement was observed relative to the other noisereduction methods. The PSNR value is calculated as the noise component divided by the MSE of the pixel values of the normal-dose and denoised images. Therefore, both insufficient denoising and excessive smoothing trigger deviations in pixel values from the original image. In this case, PSNR is degraded. PSNR values obtained by the DnCNN method were influenced by the excessive smoothing, and these results can be visually observed in Fig. 6. In contrast, because a significant improvement compared with the results of the Gaussian method can be demonstrated, the DnCNN method eliminates noise more naturally in $\mathrm{CT}$ image denoising than other methods. However, it has been observed that this oversmoothing behavior is associated with the original DnCNN in denoising very low-dose CT data. This is the same tendency as described in a previous study [8]. The CT value of the internal body was overestimated by applying the original DnCNN method (Fig. 4j). Conversely, the DnCNN_Tra was able to eliminate noise and maintain image sharpness at the $10 \%$ and $5 \%$ dose-equivalent levels; therefore, the clinical anatomical structure (hepatic sickle mesentery) could be visualized (white arrows in Fig. 5). Moreover, the CT profile agreed well with that of the normal-dose image (Fig. 4k). Therefore, SSIM and PSNR results were significantly increased relative to those of the original DnCNN. Therefore, the current denoising model, which was pre-trained on natural/synthetic images using Gaussian noise, can be updated by simulating dose-reduction images. However, at doseequivalent levels of $75 \%$ and $50 \%$, the results of the DnCNN_Tra images also exhibited excessive smoothing (red arrows in Fig. 5). This excessive smoothing triggered pixel value deviations from the original dose image; therefore, SSIM and PSNR values decreased in $75 \%$ and $50 \%$ dose-equivalent images compared to those in $10 \%$ and $5 \%$ images. Moreover, several inaccurate blackout regions occurred in the results for the 5\% doseequivalent image (red arrows in Fig. 5). Accordingly, low-dose CT with dose-reduction levels of $\leq 50 \%$ is sufficient when using the original DnCNN method, and $50 \%$ to $10 \%$ dose-equivalent images are adequate for adopting the trained DnCNN model for image denoising. Moreover, CNN-based denoising of images at dose equivalent levels of $<10 \%$ has a high risk of generating new image artifacts. Therefore, CNN-based denoising for images with dose-equivalent levels of $<10 \%$ from the original dose cannot create accurate noise reduction images. The proposed transfer learning could not have performed well at all dose reduction levels. The distribution of high-noise components is possibly significant for the training data. Moreover, residual learning of Poisson

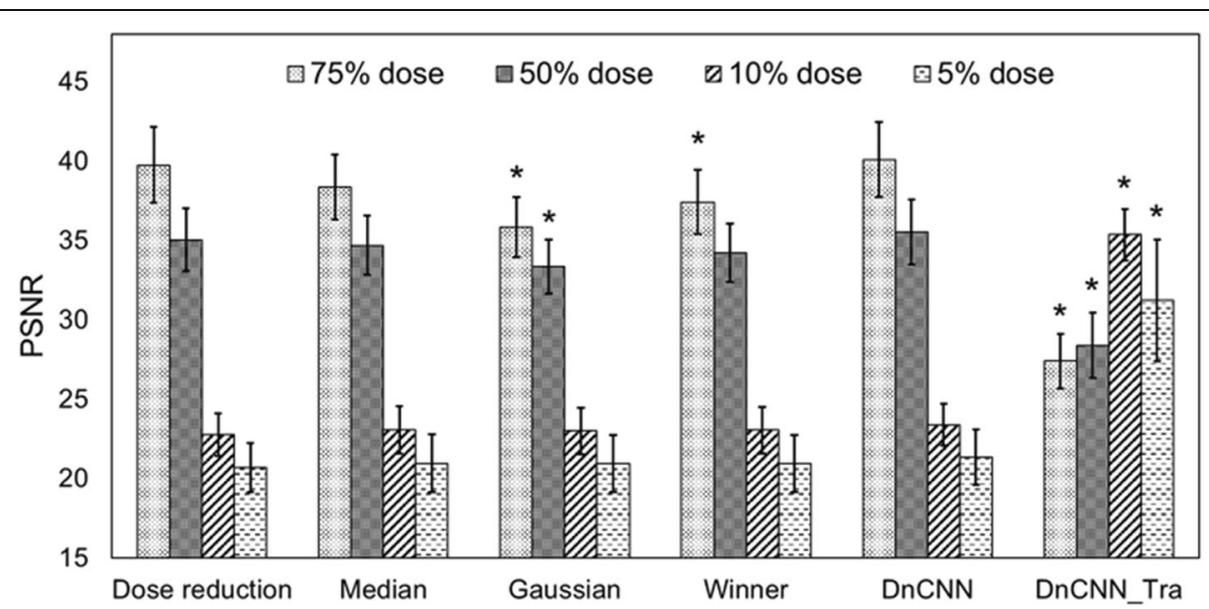

Fig. 7 PSNR results for different noise-reduction methods of each dose-reduction simulated image. * indicates $p<0.05$, which represents a significant difference in PSNR value relative to the results of the original DnCNN method 
distribution-based CT-specific noise features may have resulted in excessive smoothing. In the transfer learning process, the pre-trained learning model with prior data was maintained, and only the last fully connected layer was updated for the new data. Therefore, the CT noise became excessive, thus resulting in over-smoothing, which occurred in the high-dose region with less noise areas. The deeper the layer, the more abstract the learned feature. This can be attributed to the lack of new networks for extracting important features from training images, which is a result of the small amount of noise variation data used in training them $[18,19]$. In previous studies, the reconstruction of the learning mechanism for a specific purpose was achieved by optimizing the learning model by transfer learning [20-22]. In these studies, training data were experimentally created using human image and phantom data; therefore, the accuracy of their noise features was realistic and of high quality. Moreover, adjustments in the noise distribution of the training data and improvements in the $\mathrm{CNN}$ architecture are required to reduce noise at any dose level in clinical CT images.

The limitations of this study include the need to train and evaluate the denoising accuracy of the DnCNN method in actual low-dose exposure images. However, it is impossible to obtain CT images with the same organ positions without any misalignment obtained at various dose levels for model learning. In this study, the noiseremoval capability of the DnCNN method can be quantitatively demonstrated using unique CT noisesimulation images. In contrast, the accuracy of this CT noise simulation will influence the results of $\mathrm{CNN}$-based denoising, and realistic CT noises will increase the accuracy of the deep learning model.

\section{Conclusions}

The proposed DnCNN-based denoising method adequately denoised the CT-specific noise resulting from low-dose X-ray exposure. Furthermore, the denoising properties of the proposed method are more suitable than those of other noise-reduction methods, particularly at ultra-low-dose levels. Transfer learning with tailored DnCNN facilitated the elimination of image noise, and prevented over-smoothing at ultra-low doses. In addition, it improved image similarity by approximately $10 \%$. However, dose-equivalent levels of $<10 \%$ of the original dose could not create accurate noise reduction images. Developing a generally applicable denoising network via optimal network design and training data modification is required for appropriate noise reduction at all noise levels.

\section{Abbreviations}

$C T$ : Computed tomography; CNN: Convolutional neural network:

Dn: Denoising network; SSIM: Structural similarity index; PSNR: Peak signal-to- noise ratio; ReLU: Rectified linear units; MTF: Modulation transfer function; MSE: Mean squared error; BN: Batch normalization

\section{Acknowledgements}

We would like to thank the members of the Faculty of Health Science at Juntendo University.

\section{Authors' contributions}

$\mathrm{KU}$ conceived the study, performed analysis and interpretation of data, and drafted the manuscript; $K O, M G, Y S, S K$, and HD were involved in the study design and contributed significantly to the editing of the manuscript; All authors read and approved the final manuscript.

\section{Funding}

This work was supported by JSPS KAKENHI, No. 18 K15563.

Availability of data and materials

The datasets generated and analyzed during the current study are available in the Cancer Imaging Archive repository (https://www.

cancerimagingarchive.net/).

\section{Declarations}

\section{Competing interests}

The authors declare that they have no competing interests.

\section{Author details}

${ }^{1}$ Department of Radiological Technology, Faculty of Health Science, Juntendo University, Tokyo 113-8421, Japan. ²Department of Radiation Oncology, Faculty of Medicine, Juntendo University, Tokyo 113-8421, Japan.

${ }^{3}$ Faculty of Science and Engineering, Hosei University, Tokyo 184-8584, Japan.

Received: 31 March 2021 Accepted: 10 June 2021

Published online: 25 July 2021

References

1. Brenner DJ, Hall EJ (2007) Computed tomography-an increasing source of radiation exposure. New Engl J Med 357(22):2277-2284. https://doi.org/10.1 056/NEJMra072149

2. National Lung Screening Trial Research Team, Aberle DR, Adams AM, Berg CD, Black WC, Clapp JD et al (2011) Reduced lung-cancer mortality with low-dose computed tomographic screening. N Engl J Med 365(5):395-409. https://doi.org/10.1056/NEJMoa1102873

3. Kambadakone AR, Prakash P, Hahn PF, Sahani DV (2010) Low-dose CT examinations in Crohn's disease: impact on image quality, diagnostic performance, and radiation dose. AJR Am J Roentgenol 195(1):78-88. https://doi.org/10.2214/AJR.09.3420

4. Wang J, Li T, Lu H, Liang Z (2006) Penalized weighted least-squares approach to sinogram noise reduction and image reconstruction for lowdose X-ray computed tomography. IEEE Trans Med Imaging 25(10):12721283. https://doi.org/10.1109/TMI.2006.882141

5. Pickhardt PJ, Lubner MG, Kim DH, Tang J, Ruma JA, Rio AM et al (2012) Abdominal $C T$ with model-based iterative reconstruction (MBIR): initial results of a prospective trial comparing ultralow-dose with standard-dose imaging. AJR Am J Roentgenol 199(6):1266-1274. https://doi.org/10.2214/A JR.12.9382

6. Tian Z, Jia X, Yuan K, Pan T, Jiang SB (2011) Low dose CT reconstruction via edge-preserving total variation regularization. Phys Med Biol 56(18):59495967. https://doi.org/10.1088/0031-9155/56/18/011

7. Zhao T, McNitt-Gray M, Ruan D (2019) A convolutional neural network for ultra-low-dose CT denoising and emphysema screening. Med Phys 46(9): 3941-3950. https://doi.org/10.1002/mp.13666

8. Kim B, Han M, Shim H, Baek J (2019) A performance comparison of convolutional neural network-based image denoising methods: the effect of loss functions on low-dose CT images. Med Phys 46(9):3906-3923. https://doi.org/10.1002/mp.13713

9. Wolterink JM, Leiner T, Viergever MA, Isgum I (2017) Generative adversarial networks for noise reduction in low-dose CT. IEEE Trans Med Imaging 36(12):2536-2545. https://doi.org/10.1109/TMI.2017.2708987 
10. Zhang K, Zuo W, Chen Y, Meng D, Zhang L (2017) Beyond a gaussian denoiser: residual learning of deep CNN for image denoising. IEEE Trans Image Process 26(7):3142-3155. https://doi.org/10.1109/TIP.2017.2662206

11. Rajeev R, Samath JA, Karthikeyan NK (2019) An intelligent recurrent neural network with long short-term memory (LSTM) BASED batch normalization for medical image denoising. J Med Syst 43(8):234. https://doi.org/10.1007/ s10916-019-1371-9

12. Tian C, Fei L, Zheng W, Xu Y, Zuo W, Lin CW (2020) Deep learning on image denoising: an overview. Neural Netw 131:251-275. https://doi.org/1 0.1016/j.neunet.2020.07.025

13. Chen H, Zhang Y, Zhang W, Liao P, Li K, Zhou J et al (2017) Low-dose CT via convolutional neural network. Biomed Opt Express 8(2):679-694. https:// doi.org/10.1364/BOE.8.000679

14. Chen H, Zhang Y, Kalra MK, Lin F, Chen Y, Liao P et al (2017) Low-dose CT with a residual encoder-decoder convolutional neural network (RED-CNN). IEEE Trans Med Imaging 36(12):2524-2535. https://doi.org/10.1109/TMI.201 7.2715284

15. Clark K, Vendt B, Smith K, Freymann J, Kirby J, Koppel P et al (2013) The Cancer imaging archive (TCIA): maintaining and operating a public information repository. J Digit Imaging 26(6):1045-1057. https://doi.org/10.1 007/s10278-013-9622-7

16. Kim CW, Kim JH (2014) Realistic simulation of reduced-dose CT with noise modeling and sinogram synthesis using DICOM CT images. Med Phys 41(1): 011901. https://doi.org/10.1118/1.4830431

17. Wang Z, Bovik AC, Sheikh HR, Simoncelli EP (2004) Image quality assessment: from error visibility to structural similarity. IEEE Trans Image Process 13(4):600-612. https://doi.org/10.1109/TIP.2003.819861

18. Tang S, Yang M, Bai J (2020) Detection of pulmonary nodules based on a multiscale feature 3D U-net convolutional neural network of transfer learning. PLoS One 15(8):e0235672. https://doi.org/10.1371/journal.pone.023 5672

19. Liang $G$, Zheng $L$ (2019) A transfer learning method with deep residual network for pediatric pneumonia diagnosis. Comput Methods Prog Biomed 187:104964. https://doi.org/10.1016/j.cmpb.2019.06.023

20. Yang Y, Yan LF, Zhang X, Han Y, Nan HY, Hu YC et al (2018) Glioma grading on conventional MR images: a deep learning study with transfer learning. Front Neurosci 12:804. https://doi.org/10.3389/fnins.2018.00804

21. Gomi T, Sakai R, Hara H, Watanabe Y, Mizukami S (2019) Development of a denoising convolutional neural network-based algorithm for metal artifact reduction in digital tomosynthesis for arthroplasty: a phantom study. PLoS One 14(9):e0222406. https://doi.org/10.1371/journal.pone.0222406

22. Chow DS, Khatri D, Chang PD, Zlochower A, Boockvar JA, Filippi CG (2020) Updates on deep learning and glioma: use of convolutional neural networks to image glioma heterogeneity. Neuroimaging Clin N Am 30(4): 493-503. https://doi.org/10.1016/j.nic.2020.07.002

\section{Publisher's Note}

Springer Nature remains neutral with regard to jurisdictional claims in published maps and institutional affiliations.

\section{Submit your manuscript to a SpringerOpen ${ }^{\circ}$ journal and benefit from:}

- Convenient online submission

- Rigorous peer review

- Open access: articles freely available online

- High visibility within the field

- Retaining the copyright to your article

Submit your next manuscript at $\boldsymbol{\nabla}$ springeropen.com 